\title{
Fingerprint Intermolecular Interactions of Sodium and Potassium Complexes
}

\author{
M.Bhavya*, R.Malarvizhi \\ $P G \&$ Research Department of Chemistry Seethalakshmi Ramaswami College Affiliated to Bharathidasan \\ University Tiruchirappalli-620002, Tamil Nadu, India
}

*Corresponding Authors: M.Bhavya, PG \& Research Department of Chemistry Seethalakshmi Ramaswami College Affiliated to Bharathidasan University Tiruchirappalli-620002, Tamil Nadu, India

\begin{abstract}
The complex of sodium and potassium salts is characterized by using UV-Visible, FT-IR, NMR,
Single- crystal X-ray diffraction, Hirshfeld surfaces, and Fingerprint plots. The crystal belongs to the monoclinic system and p121/n1, p21/n space group. The corresponding peaks for chlorine atoms are absent in the NMR spectra of the complexes. The small red spot in Hirshfeld surfaces indicates the presence of $C$ $H . . . \pi$ interactions in the sodium complex. Absence of Metal...Metal interactions were also observed in both sodium and potassium complex. Fingerprint plots of Hirshfeld surfaces were used to detect and examine the percentage of hydrogen- bonding interactions.
\end{abstract}

Keywords: 1, 3- dimethyl barbituric acid, sodium methoxide, potassium methoxide, Hirshfeld surfaces, and fingerprint plots.

\section{INTRODUCTION}

A recurring theme in very recent publications devoted to intermolecular interactions in solids. To consider two or more structures i.e., similar or dissimilar, two are involved in these interactions. a) identification of the core structural features b) evaluation of the extent of their resemblance. Hirshfeld surfaces encircle the molecules surrounded by the crystals. ${ }^{1}$ The locus of points in Hirshfeld surfaces are those for which the ratio of electron density contributions computed from a sum of atoms in a test molecule with spherical scattering factors to the electron density contribution determined from all of the atoms. Hirshfeld surfaces are typically plotted at a ratio of 0.5 , that place where the contribution to the electron density from a molecule is equal to the contribution from all the other molecules. As such, Hirshfeld surfaces are reactive to intermolecular interactions and can be elaborated by plotting on them any scalar function that can be devised from the crystal structure or the surface itself. Predominantly informative are Hieshfeld surfaces on which are plotted the distance to the nearest atom within the surface or external to it.

The 2D plots are a novel illustration representation of all the intermolecular interactions at the same time and are exclusive for a given crystal structure and polymorph. In this paper, we reported crystal structure characterized by using Single- crystal X-ray diffraction (XRD), UV-visible, FT-IR, NMR, Hirshfeld surfaces, Fingerprint analysis.

\section{EXPERIMENTAL METHODS}

\subsection{Synthesis of Molecular Salts}

1-Chloro2,4-dinitrobenzene (DNCB) is dissolved in 30ml of ethanol is mixed with 1,3-dimethyl barbituric acid in $30 \mathrm{ml}$ of ethanol. Then added Sodium/potassium methoxide in $30 \mathrm{ml}$ of ethanol and shaken for 2-3 hrs. The excess ethanol be removed by distillation also the remaining solution was kept for one week when red- colored crystals separated. These crystals are recrystallized from distilled ethanol.

\subsection{Characterization Techniques}

The UV-Visible spectra were obtained by using Shimadzu UV/VIS 1800 spectrophotometer. The IR spectra were recorded by using Perkin-Elmer RXI infrared spectrophotometer as KBr pellets. The NMR 
spectra data were obtained from Bruker DRX - $500 \mathrm{MHz}$ spectrophotometers with (DMSO-d6) is used as a solvent and tetramethylsilane is used as the internal reference. A single-crystal X-ray diffraction study has the dimensions $0.15 \mathrm{~mm} \times 0.26 \mathrm{~mm} \times 0.28 \mathrm{~mm}$ was used for structural determination. The frames have been integrated with the Bruker SAINT Software package by using a narrow-frame algorithm.

\subsection{Thin Layer Chromatographic Studies}

The study was carried out to check the purity of the isolated molecules. Silica gel was used as an adsorbent. The slurry of silica gel was made with chloroform and coated uniformly on a TLC plate. The complex solution was prepared in pure absolute ethanol and spotted on the plate. The eluent used was the mixture of tertiary butyl alcohol and ethyl acetate.

\section{RESULTS AND DISCUSSION}

\subsection{Crystal Structure X-Ray Diffraction}

\subsubsection{Crystal Structure of Sodium Salt}

The amorphous solid of sodium salt is crystallized from ethanol. The sodium cations are bridged through an oxygen atom of 1-chloro-2, 4-dinitro benzene as shown in fig 1. In barbiturate residue bond lengths, bond angles are well-matched with that of barbiturate ion $^{2}$ and it shows the delocalization of negative charge. The crystal data represent selected bond angles, bond distances of sodium salt. According to X-ray crystallographic data, the isolated molecule is a monoclinic system. The unit cell parameters (Table 1) having P121/n1 space group $a=7.3269(5) \AA, b=19.9241(13) \AA, c=11.5431(9) \AA$, $V=1665.1(2) \AA^{3} ; Z=4$.

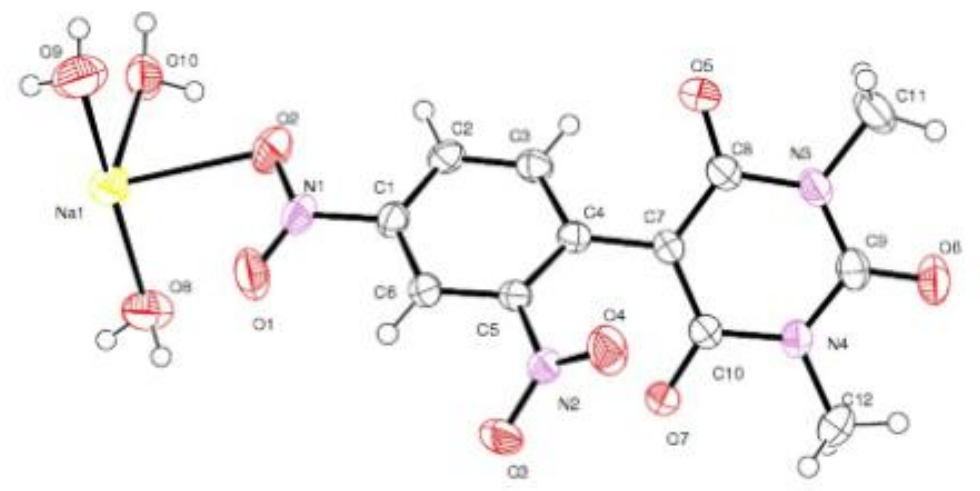

Fig1. ORTEP View of sodium Complex

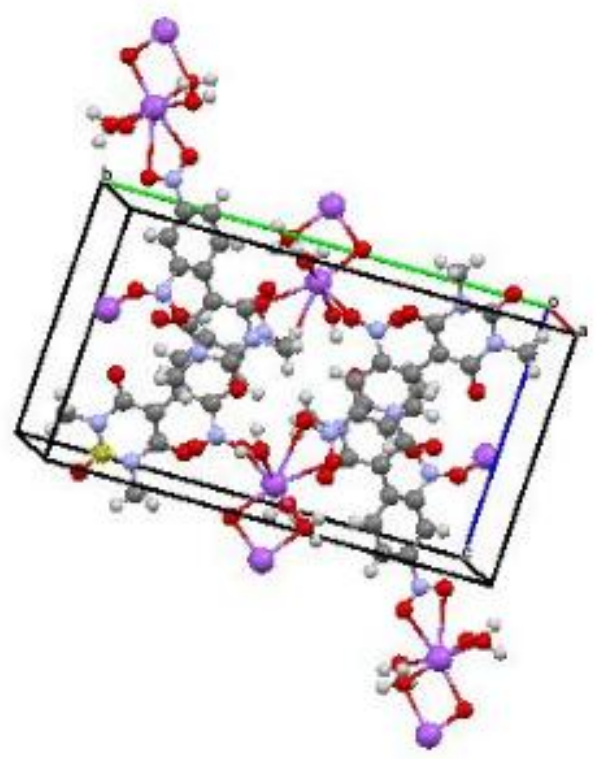

Fig1a. Packing View of sodium Complex 


\subsubsection{Crystal structure of potassium salt}

The structure of the potassium complex with DNCB, the crystals have been confirmed based on unitcell contents. The crystal studies indicate that potassium cations are 8-coordinated and are bridged by an oxygen atom of 1,3-dimethyl barbituric acid (Fig 2). The K-O bond length range is 2.778-3.8699 (Shrivastava \& Speakman, 1961). The Single-crystal XRD results have shown that the potassium salt of the present investigation possesses an organometallic polymeric association. The crystal data represent selected bond distances, bond angles of potassium salt. The molecule is a monoclinic crystal system having a P21/n space group and cell parameters are $a=11.3166(7) \AA, b=7.2477 \AA$, $\mathrm{c}=20.3511(11) \AA ; \mathrm{Z}=4$ as shown in Table 2 .

Table1. Crystal data

\begin{tabular}{|c|c|c|}
\hline Chemical formula & \multicolumn{2}{|l|}{$\mathrm{C}_{12} \mathrm{H}_{15} \mathrm{~N}_{4} \mathrm{NaO}_{10}$} \\
\hline Formula weight & \multicolumn{2}{|l|}{$398.27 \mathrm{~g} / \mathrm{mol}$} \\
\hline Temperature & \multicolumn{2}{|l|}{$296(2) \mathrm{K}$} \\
\hline Wavelength & \multicolumn{2}{|c|}{$0.71073 \AA$} \\
\hline Crystal size & \multicolumn{2}{|c|}{$0.150 \times 0.260 \times 0.280 \mathrm{~mm}$} \\
\hline Crystal habit & \multicolumn{2}{|c|}{ clear dark brown Rectangular } \\
\hline Crystal system & \multicolumn{2}{|c|}{ Monoclinic } \\
\hline Space group & \multicolumn{2}{|l|}{$\mathrm{P} 121 / \mathrm{n} 1$} \\
\hline \multirow[t]{3}{*}{ Unit cell dimensions } & $\mathrm{a}=7.3269(5) \AA$ & $\alpha=90^{\circ}$ \\
\hline & $\mathrm{b}=19.9241(13) \AA$ & $\beta=98.822(4)^{\circ}$ \\
\hline & $\mathrm{c}=11.5431(9) \AA$ & $\gamma=90^{\circ}$ \\
\hline Volume & $1665.1(2) \AA^{3}$ & \\
\hline $\mathbf{Z}$ & \multicolumn{2}{|l|}{4} \\
\hline Density (calculated) & \multicolumn{2}{|l|}{$1.589 \mathrm{~g} / \mathrm{cm}^{3}$} \\
\hline Absorption coefficient & \multicolumn{2}{|l|}{$0.160 \mathrm{~mm}^{-1}$} \\
\hline $\mathbf{F}(\mathbf{0 0 0})$ & \multicolumn{2}{|l|}{824} \\
\hline
\end{tabular}

Table2. Crystal data

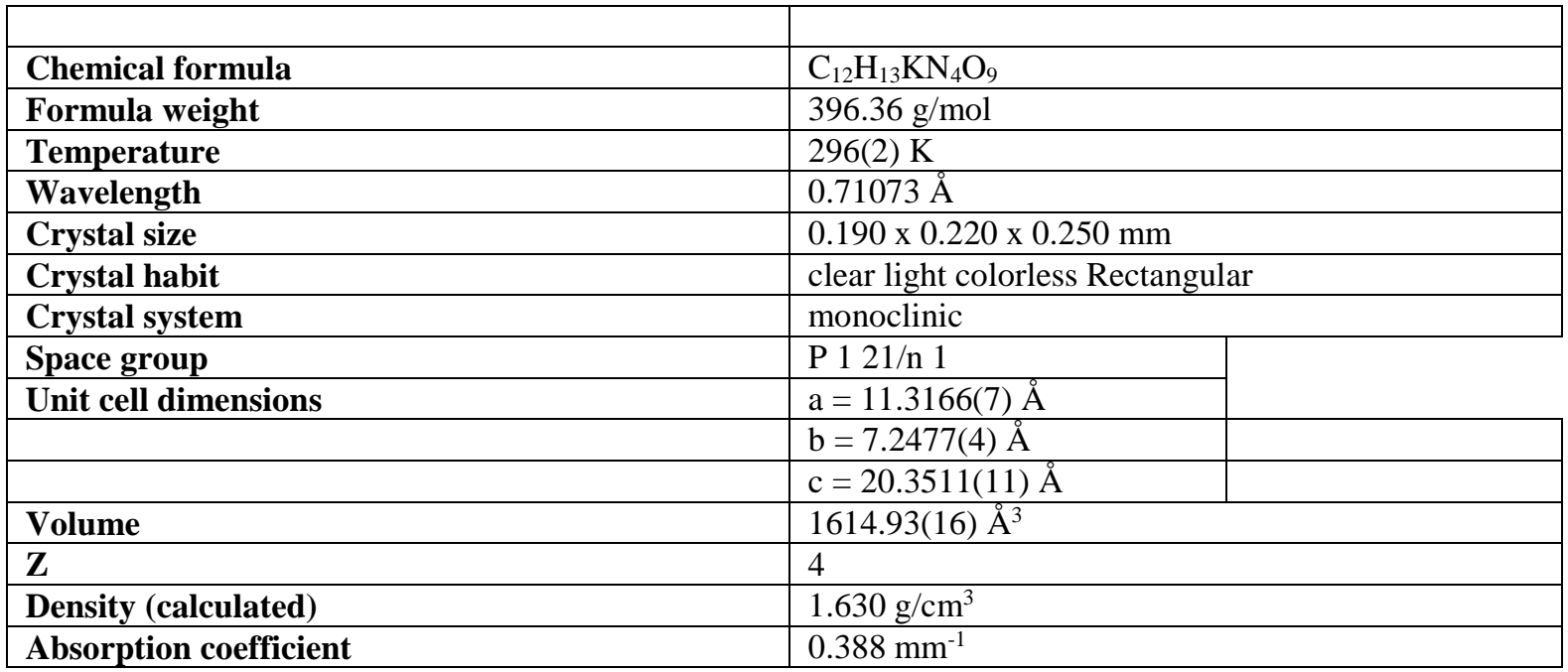

Table3.

\begin{tabular}{|l|l|}
\hline Interactions & Sodium complex (\%) \\
\hline O-H & $25.3 \%$ \\
\hline H-H & $23.3 \%$ \\
\hline C-O & $1.3 \%$ \\
\hline O-O & $2.9 \%$ \\
\hline C-C & $2.1 \%$ \\
\hline C-H & $6.6 \%$ \\
\hline N-O & $\mathbf{0 . 4 \%}$ \\
\hline C-N & $\mathbf{0 . 6 \%}$ \\
\hline N-H & $1.8 \%$ \\
\hline N-N & $0.6 \%$ \\
\hline
\end{tabular}


Table4. Quantitative measures of Hirshfeld surfaces for sodium and potassium complexes

\begin{tabular}{|l|l|l|l|l|}
\hline Complex & $\mathbf{V}_{\mathbf{H}}\left(\mathbf{\AA}^{\mathbf{3}}\right)$ & $\mathbf{S}_{\mathbf{H}}\left(\mathbf{\AA}^{\mathbf{2}}\right)$ & $\mathbf{G}$ & $\mathbf{\Omega}$ \\
\hline Sodium complex & 405.63 & 395.43 & 0.670 & 0.35 \\
\hline Potassium complex & 390.74 & 377.55 & 0.685 & 0.268 \\
\hline
\end{tabular}

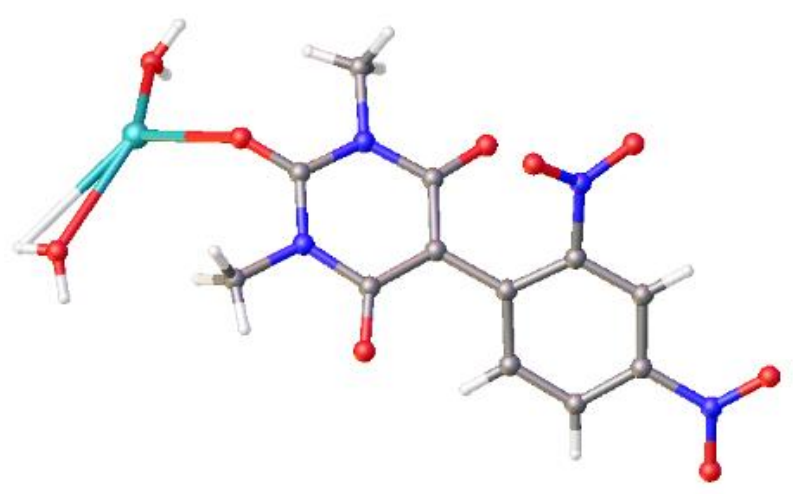

Fig2. ORTEP of potassium Complex

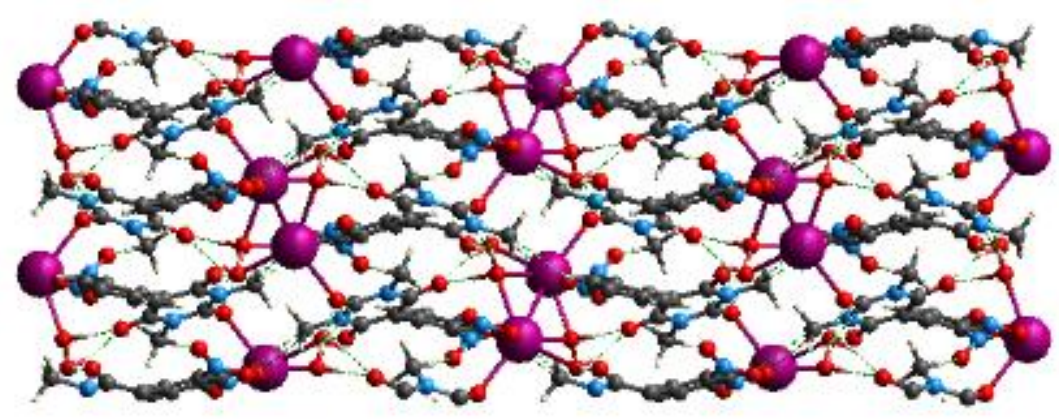

Fig2a. Packing View of potassium Complex

\subsection{UV-Visible Analysis}

In the UV-Vis spectrum, the two peaks are formed with $\lambda$ max values of $287.30 \mathrm{~nm}, 484.34 \mathrm{~nm}$, and $245.93 \mathrm{~nm}, 446.86 \mathrm{~nm}$ (Fig 3,3a)

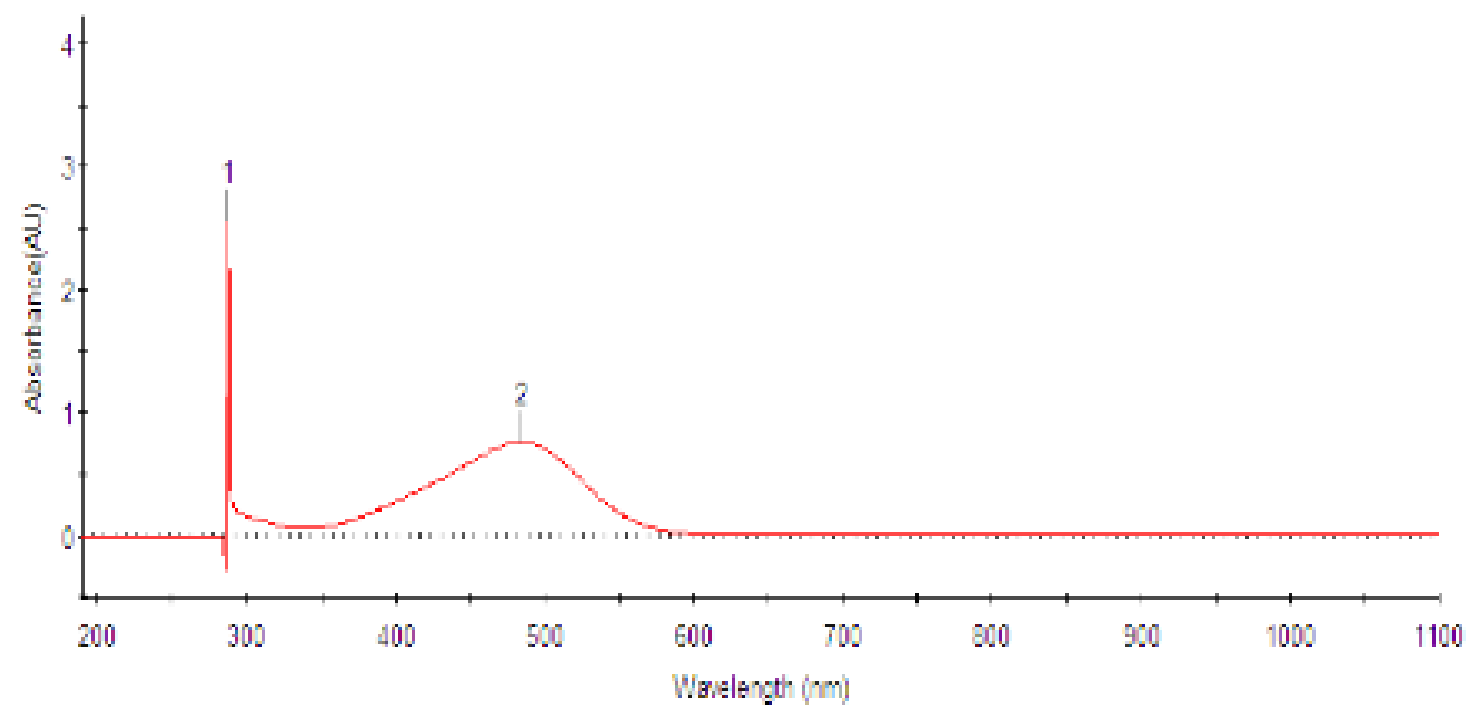

Fig-3: UV-Visible Spectrum of sodium Complex 


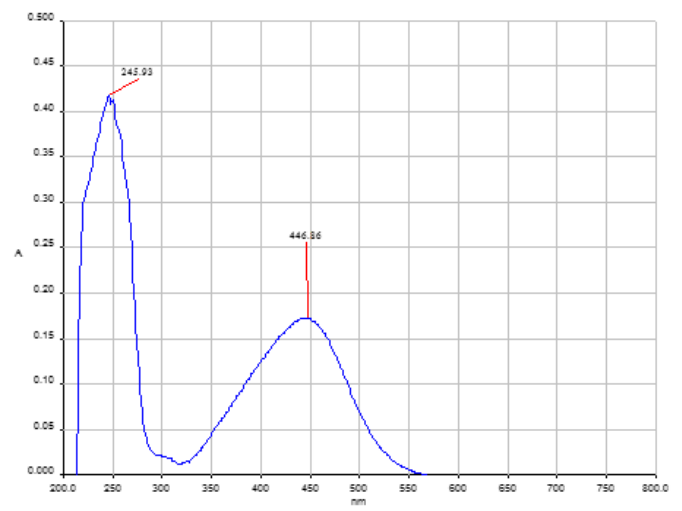

Fig-3a: UV-Visible Spectrum of potassium Complex

\subsection{FT-IR Analysis}

$\mathrm{NO}_{2}$ asymmetric and symmetric stretching frequency is $1536 \mathrm{~cm}^{-1}, 1532 \mathrm{~cm}^{-1}$ and $1331 \mathrm{~cm}^{-1}, 1327 \mathrm{~cm}^{-}$ ${ }^{1}$ as shown in fig 4,4a. Here, the $\mathrm{C}-\mathrm{Cl}$ band is absent in the synthesized molecule The broadband observed between $\sim 3600-2100 \mathrm{~cm}^{-1}$ is characteristic of amine salt ${ }^{3}$. Carbonyl stretching frequency of 1,3-diethyl barbituric acid $1680 \mathrm{~cm}^{-1}$ and $1670 \mathrm{~cm}^{-1}$ during the formation of the complex.

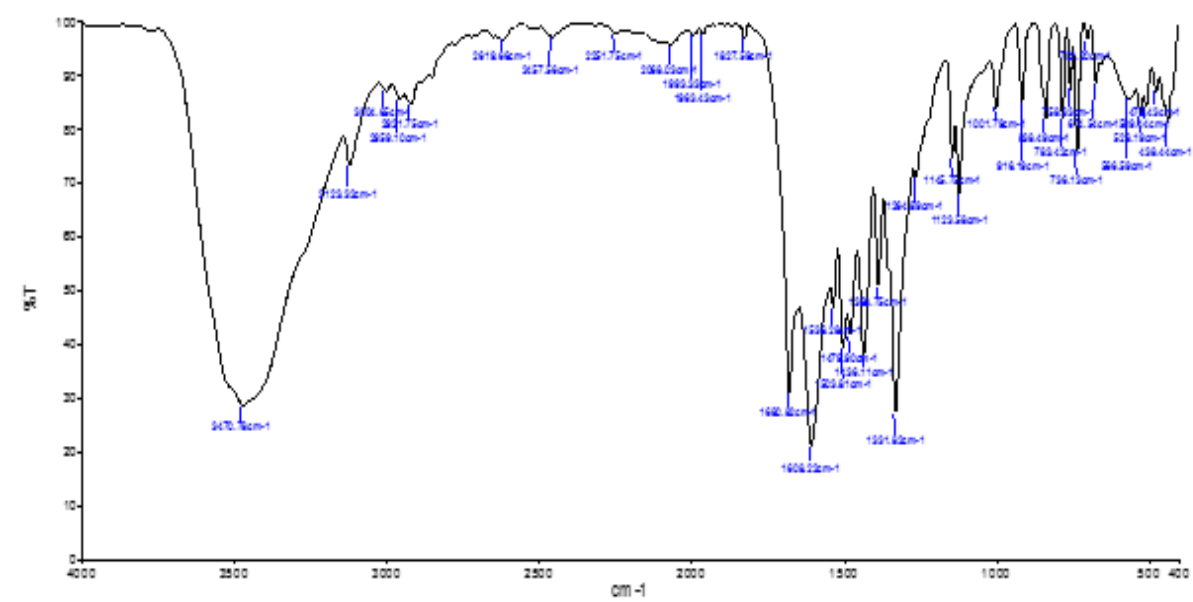

Fig -4: FT-IR Spectrum of sodium Complex

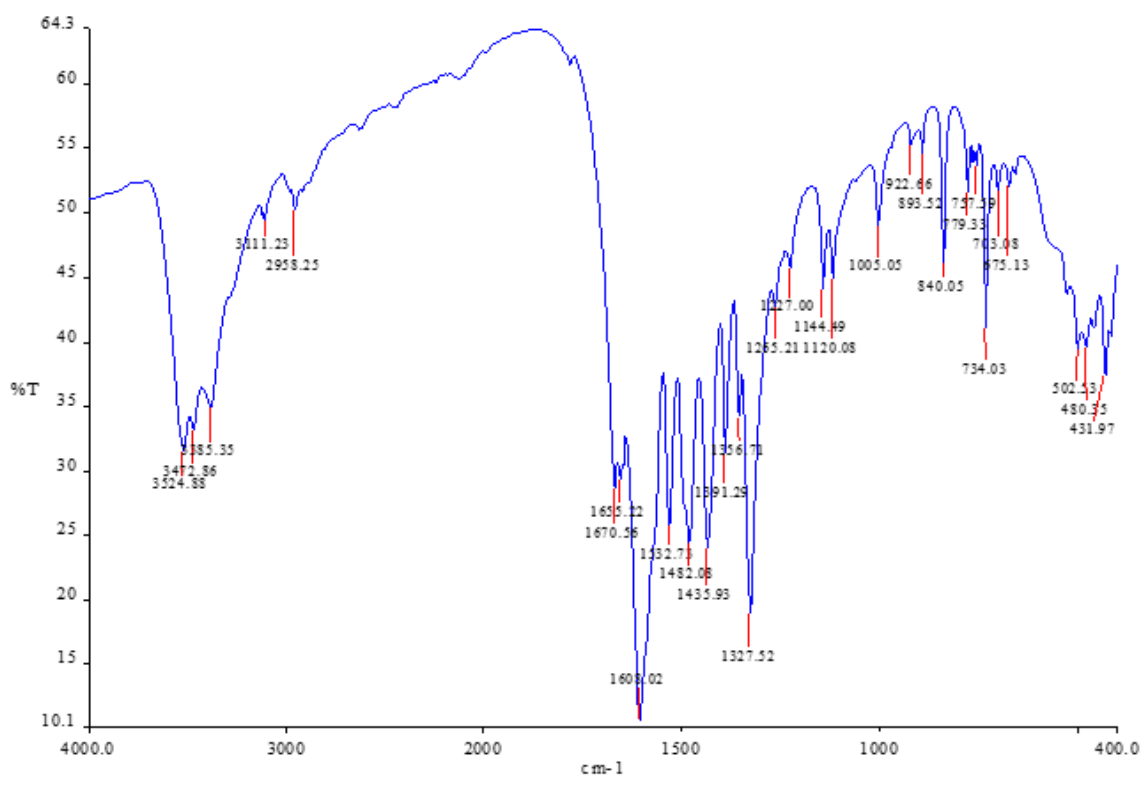

Fig -4a: FT-IR Spectrum of potassium Complex 


\subsection{NMR Analysis}

${ }^{1} \mathrm{H}$ NMR spectra are depicted in fig 5, 5a. This shows two peaks characteristics of the ring protons, one at $\delta 8.1 \mathrm{ppm}(\mathrm{S}, 2 \mathrm{H})$ and another at $\delta 8.3 \mathrm{ppm}(\mathrm{S}, 1 \mathrm{H})$ are observed. The peak corresponding to six methyl protons appears at $\delta 3.1 \mathrm{ppm} .{ }^{13} \mathrm{C}$ NMR spectra of molecular salts are presented in fig $6,6 \mathrm{a} .{ }^{13} \mathrm{C}$ NMR spectrum indicates ten different carbon environments in the molecule. The peaks at $141.5 \mathrm{ppm}$ and $87.1 \mathrm{ppm}$ represent the formation of $\mathrm{C}=\mathrm{C}$.

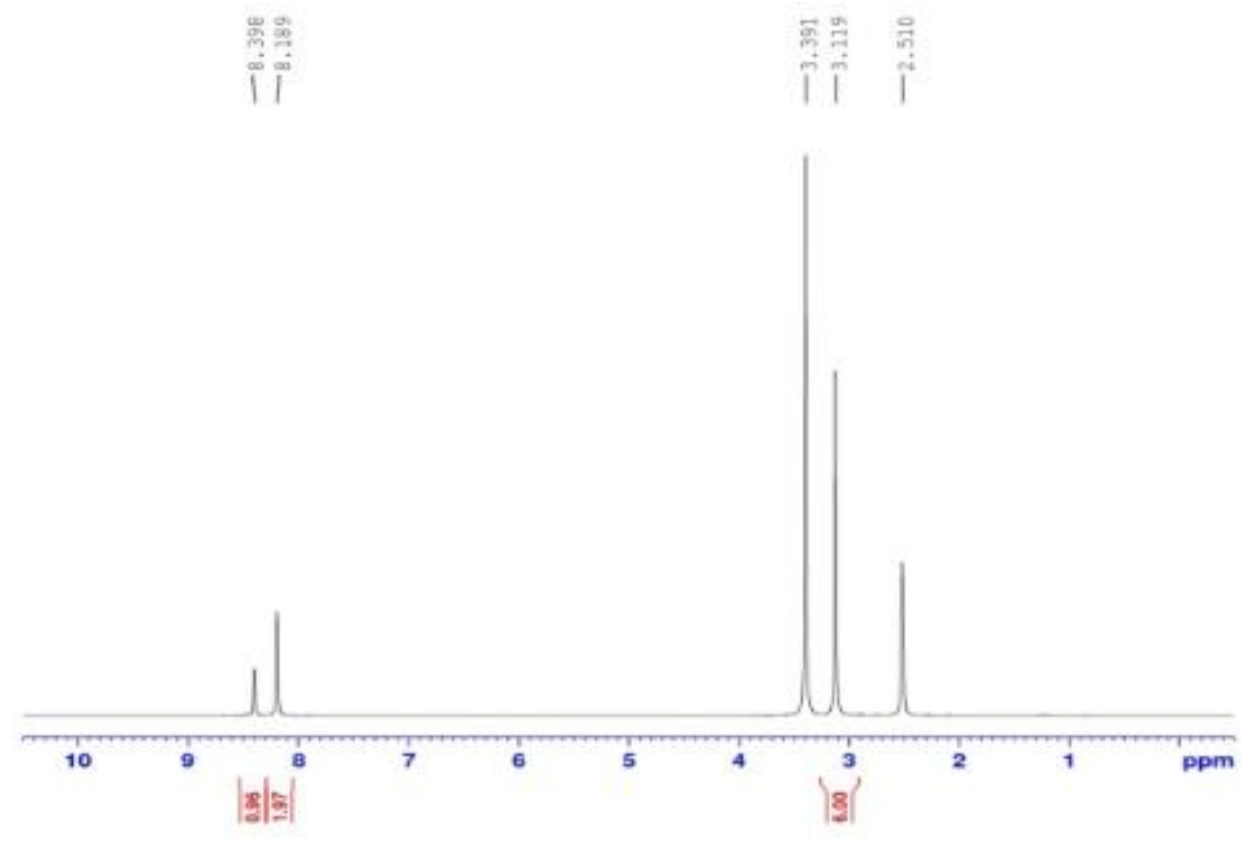

Fig- 5: ${ }^{1}$ H-NMR Spectrum of sodium Complex

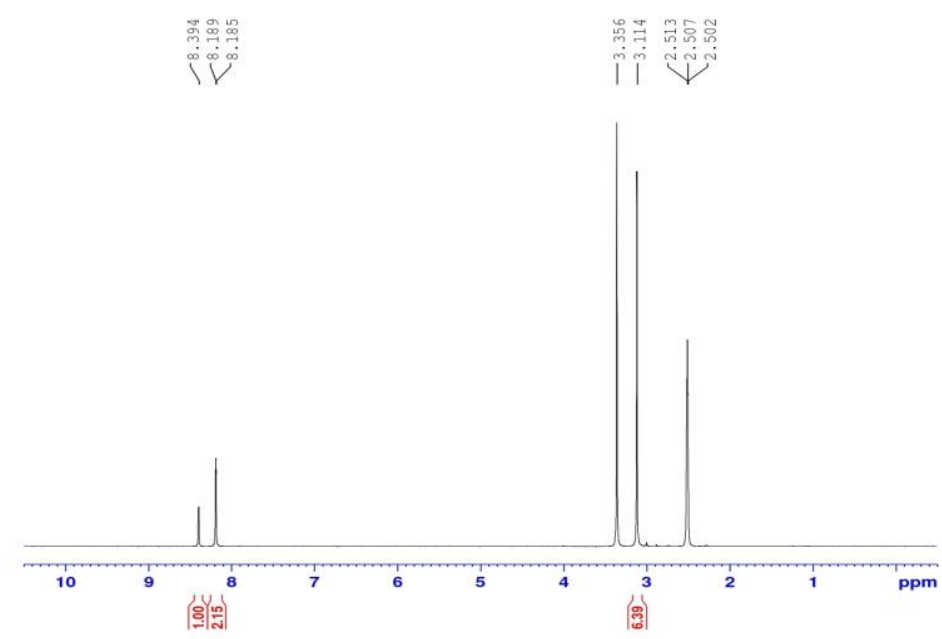

Fig- 5a: ${ }^{1}$ H-NMR Spectrum of Potassium Complex

\subsection{Hirshfeld Surface Calculations}

Hirshfeld surface analysis is a progressive technique used for measuring intermolecular interactions by consideration of the interactions throughout the whole molecule. The Hirshfeld surface has been mapped over d norm, di, de, shape index, curvedness (Mckinnon et al., 2004). Molecular Hirshfeld surface calculations ${ }^{5-7}$ have been performed by using the crystal explorer 3.1 programs. When CIF files are entered into the Crystal Explorer Program, all bond lengths to hydrogen are automatically modified to typical standard neutron values ${ }^{7}$ i.e.,

$\mathrm{C}-\mathrm{H}=1.083 \AA$, N-H=1.009 $\AA$ and $\mathrm{O}-\mathrm{H}=0.983 \AA$. For comparison of intermolecular interactions in the crystal structures, the Hirshfeld surfaces have been mapped with normalized contact distances $\left(\mathrm{d}_{\text {norm }}\right)$. 
The $\mathrm{d}_{\text {norm }}$ parameter is based on di. di is the distance from the surface to the nearest atom in the molecule itself, de is the distance from the surface to the nearest atom in another molecule, and Van der Waals radii of the corresponding atoms $\left(r_{i}{ }^{\mathrm{Vdw}}\right.$ and $\left.\mathrm{r}_{\mathrm{e}}{ }^{\mathrm{Vdw}}\right)$.

The $\mathrm{d}_{\text {norm }}$ values are employing a red-blue-white color scheme, where red regions represent closer contacts and negative $\mathrm{d}_{\text {norm }}$ value; blue regions represent longer contacts and a positive $\mathrm{d}_{\text {norm }}$ value and white regions represent the distance of contacts exactly corresponding to the Van der Waals separation with $d$ norm value of zero ${ }^{6-8}$. The negative $d_{\text {norm }}$ value indicates the sum of di and de is shorter than the sum of the relevant Van der Waals radii, which is considered to be the closest contact and is visualized as red color in the Hirshfeld surfaces. Hirshfeld surfaces also enabling rapid computation of molecular volume $\left(\mathrm{V}_{\mathrm{H}}\right)$, surface area $\left(\mathrm{S}_{\mathrm{H}}\right)$ and two other descriptors of global shape also have been calculated i) globularity $(\mathrm{G})$ and ii) asphericity $(\Omega)$. Globularity ${ }^{9}(\mathrm{G})$ is a measure of the degree to which the surface area differs from that of a sphere of the same value volume. $\mathrm{G}$ will be 1.0 for a sphere, and progressively less than one becomes more structured. Asphericity $(\Omega)^{10,11}$ is a measure of anisotropy and when applied to the positions of atoms in the molecules.

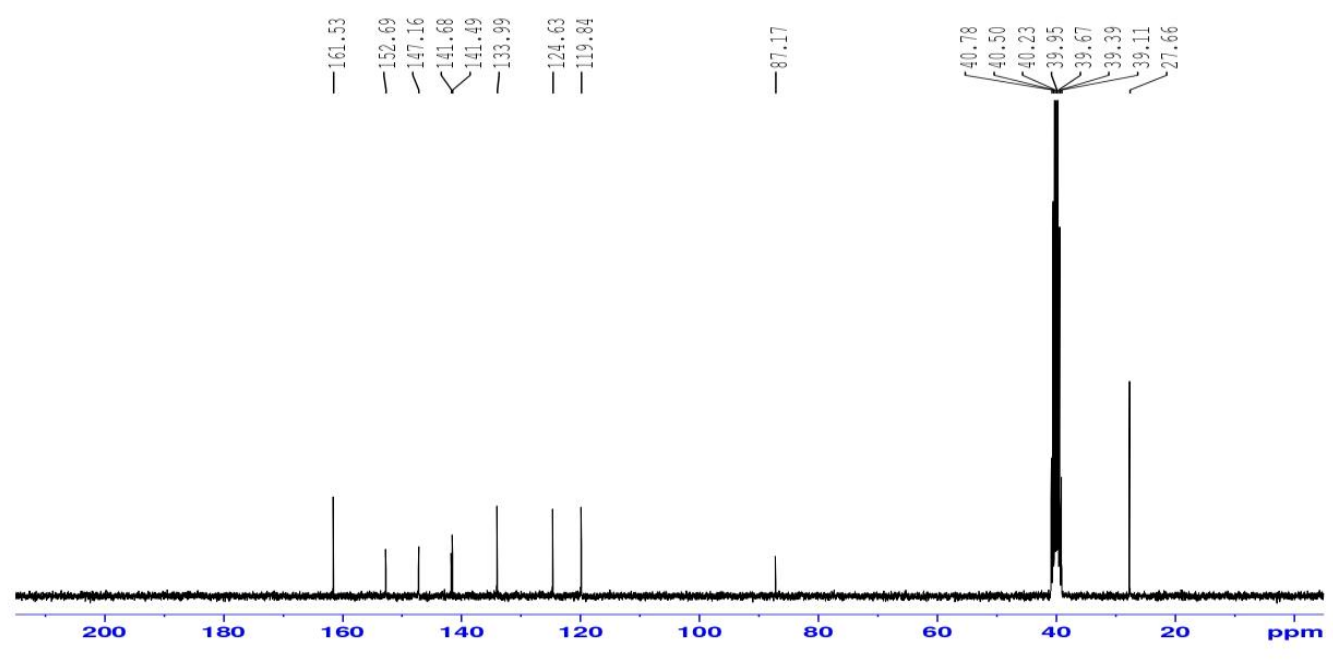

Fig -6: ${ }^{13} \mathrm{C}$ NMR Spectrum of sodium complex

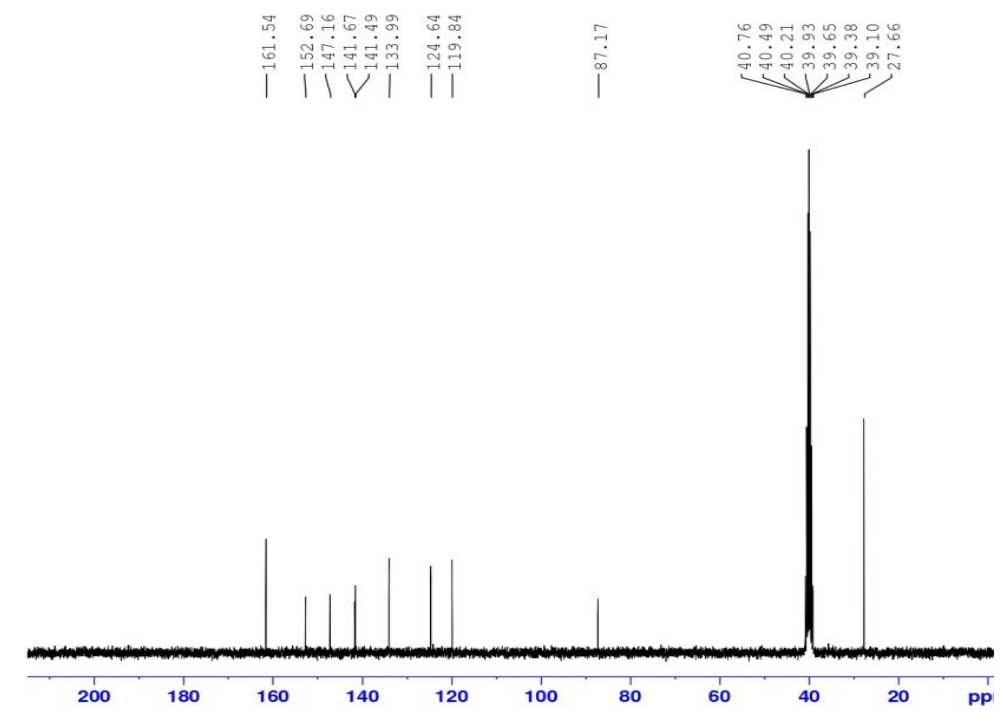

Fig -6a: ${ }^{13} \mathrm{C}$ NMR Spectrum of potassium complex

\subsection{Hirshfeld Surfaces of Sodium Complex}

The large and red spot on the 3D Hirshfeld surfaces shows those close- contact interactions, responsible for corresponding hydrogen bond contacts. Fig 7 represents Hirshfeld surfaces in various forms. The 
small red spots on surfaces indicate that $\mathrm{C}-\mathrm{H} . . . \pi$ interactions. By using 2D fingerprint plots, many interactions can be separated from others that overlap in full fingerprint plots (Fig 8). There is a C...C interaction of sodium salt $(2.1 \%)$. From this sodium salt, the $\mathrm{O} \ldots \mathrm{H}$ interactions exhibit a more significant contribution (25.3\%) compared to the total Hirshfeld surfaces. The N...O, C...N, N...H, and $\mathrm{N}$... N hydrogen bonding interactions do not exhibit significant contributions of total Hirshfeld surfaces. Metal...Metal (M...M) interactions are not observed in sodium complex (Table 3). Globularity value of sodium salt is 0.670 (Table 4 ) and then surface property statistics are listed (Table 5).
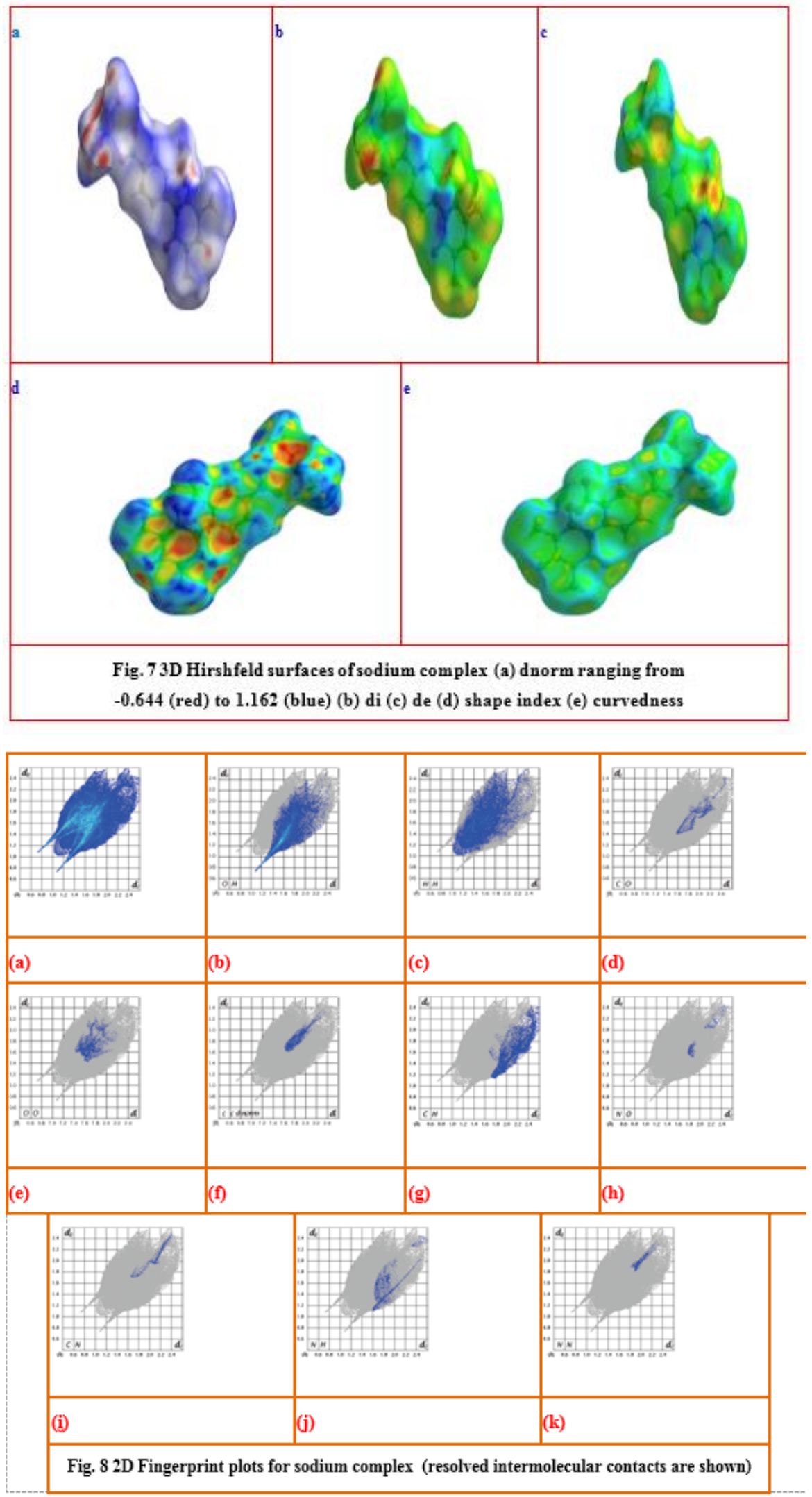


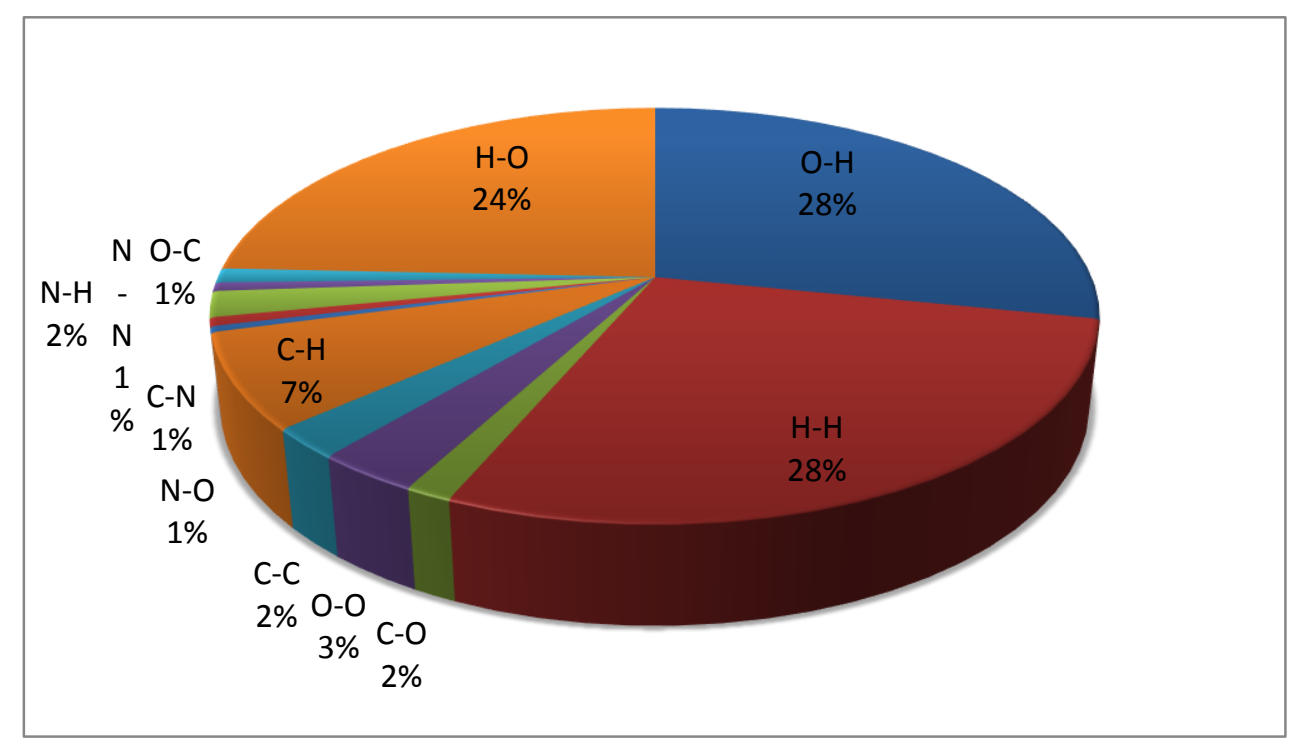

Pie chart for sodium complex

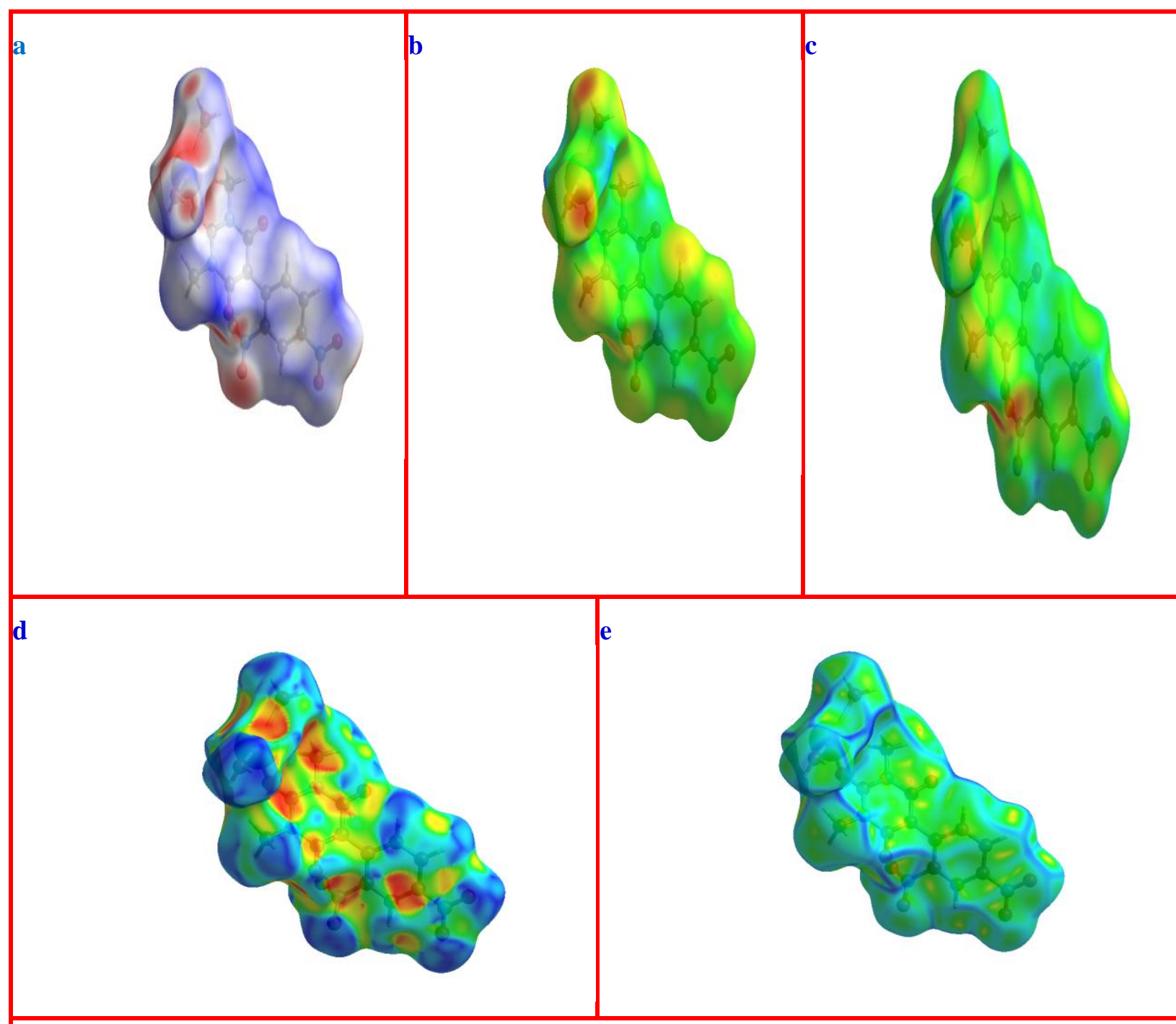

Fig. 93D Hirshfeld surfaces of potassium complex (a) dnorm ranging from $\mathbf{- 0 . 6 6 3}$ (red) to 1.195 (blue) (b) di (c) de (d) shape index (e) curvedness 


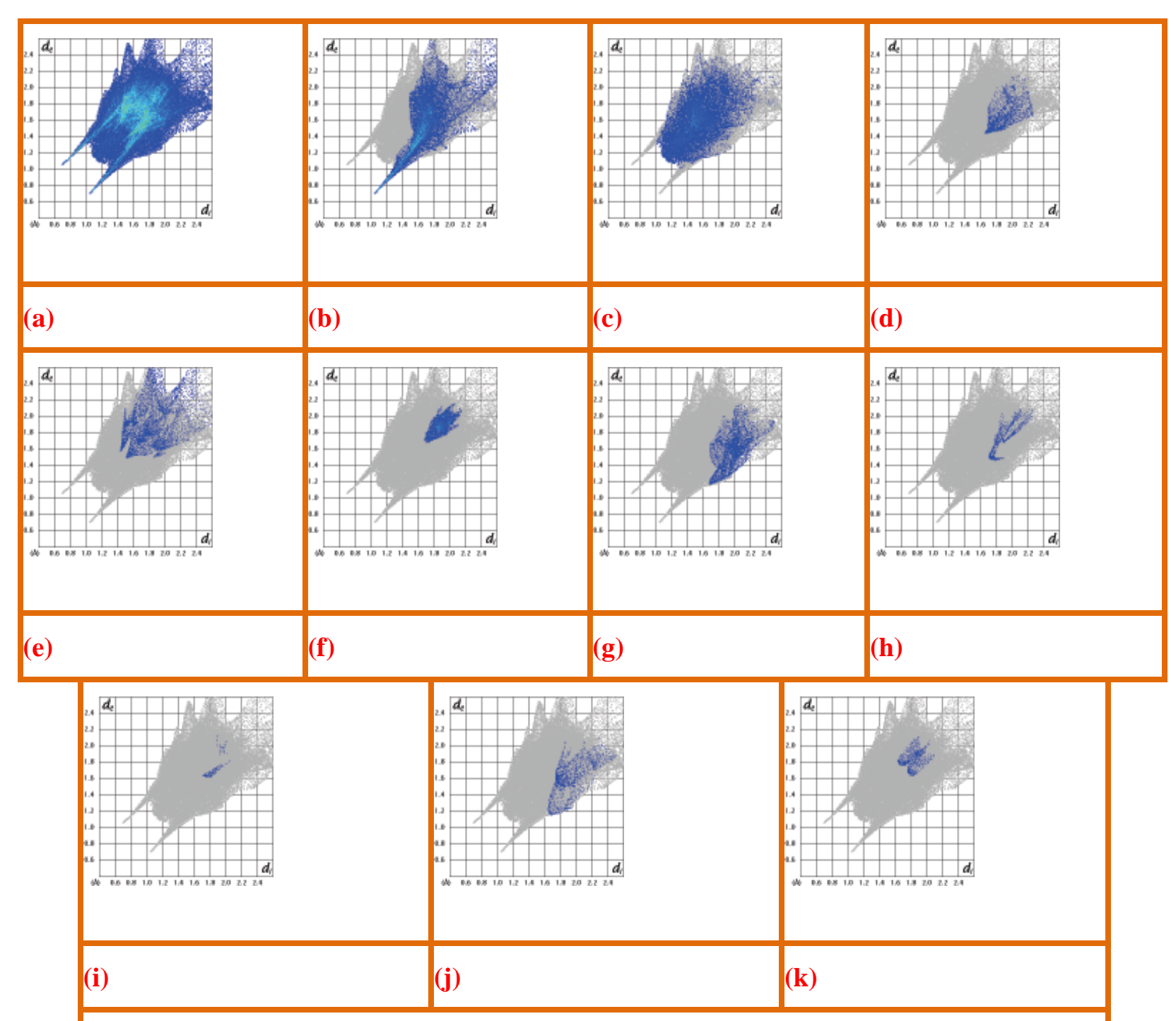

Fig. 10 2D Fingerprint plots for potassium complex (resolved intermolecular contacts are shown)

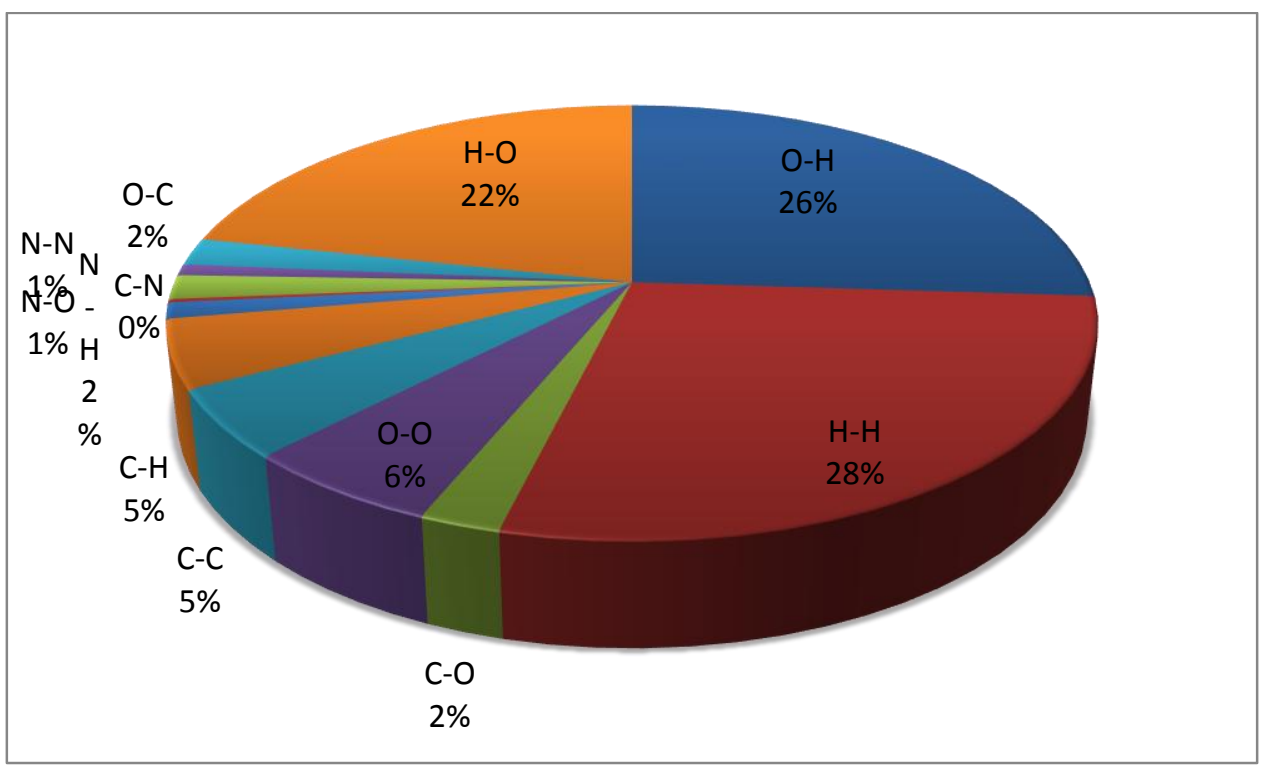

Pie chart for potassium complex 


\subsection{Hirshfeld Surface Analysis of Potassium Complex}

The 3D Hirshfeld surfaces and 2D fingerprint plots of potassium complex as shown in fig $9 \& 10$. Quantitative measures and molecular interactions of Hirshfeld surfaces are listed in Tables $4 \& 6$. From molecular interactions, the percentage of $\mathrm{O} \ldots \mathrm{H}(21.7 \%)$ hydrogen bonding interactions appear as a sharp spike in the 2D fingerprint plots. C...H, C..O, N...H, C...N and N..O (0.2-4.2\%) interactions are very small spikes in total Hirshfeld surfaces of potassium complex. The C...C/C...H interactions in 2D fingerprint contribute $4.2 \%$ and gives asymmetric pair of wings. Nitrogen interactions contact contribution amounts $0.7 \%$. The Hirshfeld surface of the potassium complex shows the percentage of contributions i.e., the number of molecular interactions for a variety of contacts. The de and di combination provides a summary of intermolecular interactions. Metal-Metal interactions are not observed moreover K...O interaction appears as very short spikes contributing 4.9\%. Globularity value is less than one as the molecular surface becomes more structured and the value is 0.685 . Surface property statistics are mentioned below (Table 7).

Table5. Surface property statistics of sodium complex

\begin{tabular}{|c|c|c|c|c|c|c|c|c|c|c|c|}
\hline \begin{tabular}{|l|l|} 
Surface & Property \\
\end{tabular} & Units & Min & Max & Mean & Mean+ & Mean- & $\mathbf{P i}$ & Sigma+ & Sigma- & SigmaT & nu \\
\hline Hirshfelddnorm & & -0.6439 & 91.1622 & 0.3289 & 0.4129 & -0.2251 & 0.2479 & 90.061245 & 50.027111 & 0.088356 & 50.2127 \\
\hline Hirshfelddi & $\AA$ & 0.7324 & 2.6588 & 1.6264 & 1.6264 & nan & 0.2349 & 90.088619 & 9 nan & nan & nan \\
\hline Hirshfeldde & & $\AA 0.7325$ & 2.6024 & 1.6469 & 1.6469 & $\operatorname{nan}$ & 0.2362 & 20.086129 & 9 nan & nan & nan \\
\hline $\begin{array}{r}\text { Hirshfeld } \begin{array}{c}\text { Shape } \\
\text { index }\end{array} \\
\end{array}$ & & -0.9942 & 20.9983 & 0.2408 & 0.5557 & -0.4520 & 0.4532 & 20.053950 & 0.0621963 & 30.115913 & 30.2488 \\
\hline HirshfeldCurvedness & & -3.8652 & 20.8387 & -0.9418 & 0.1359 & -0.9565 & 50.4370 & 00.027146 & 0.277452 & 0.304598 & 80.0812 \\
\hline
\end{tabular}

Table 6

\begin{tabular}{|l|l|}
\hline Interactions & Potassium complex (\%) \\
\hline O-H & $21.7 \%$ \\
\hline H-H & $23.2 \%$ \\
\hline C-O & $2.0 \%$ \\
\hline O-O & $\mathbf{5 . 0 \%}$ \\
\hline C-C & $\mathbf{4 . 2 \%}$ \\
\hline C-H & $\mathbf{4 . 2 \%}$ \\
\hline N-O & $\mathbf{1 . 0 \%}$ \\
\hline C-N & $\mathbf{0 . 2 \%}$ \\
\hline N-H & $1.5 \%$ \\
\hline N-N & $0.7 \%$ \\
\hline
\end{tabular}

Table7. Surface property statistics of potassium complex

\begin{tabular}{|c|c|c|c|c|c|c|c|c|c|c|}
\hline \begin{tabular}{|l|l} 
Surface & Property \\
\end{tabular} & Units Min & Max & Mean & Mean+ & Mean- & $\mathbf{P i}$ & Sigma+ & Sigma- & SigmaT & nu \\
\hline Hirshfeld dnorm & -0.6630 & 1.1955 & 50.2344 & 0.3575 & -0.2341 & 0.2479 & 0.045453 & 0.025811 & 0.071264 & 0.2310 \\
\hline Hirshfeld di & 0.7000 & 2.9437 & 71.6566 & 1.6566 & nan & 0.2289 & 0.090061 & lnan & $\operatorname{nan}$ & nan \\
\hline Hirshfeld de & 0.7053 & 2.7471 & 1.6802 & 1.6802 & nan & 0.2205 & 0.079435 & 5 nan & nan & nan \\
\hline & A & & & & & & & & & \\
\hline Hirshfeld $\mid \mathrm{S}$ & -0.9970 & 0.99 & 40.2456 & 0.5650 & -0.4615 & 0.4613 & 0.0 & & 0.1 & 0.2495 \\
\hline Hirshfeld Curvedness & -3.4825 & 0.3994 & -0.941 & 80.1074 & -0.9545 & 0.4168 & 0.006866 & 50.249085 & 0.255952 & 0.0261 \\
\hline
\end{tabular}

4. CONCLUSION

A sodium and potassium salts of carbanionic sigma complexes were synthesized and confirmed from the single- crystal X-ray diffraction technique. Both salts are monoclinic. The Hirshfeld surface is visualized by intermolecular interactions. Various kinds of interactions are seen in Hirshfeld surface analysis. Fingerprint plots are confirm the types and quantities of interactions in these complexes. Metal...Metal interactions are absent in sodium and potassium complexes of Hirshfeld surfaces.

\section{ACKNOWLEDGMENT}

I express my wholehearted gratitude to my eminent Research Supervisor Dr.R. Malarvizhi, Department of Chemistry, for scientific advice, knowledge sharing and many insightful discussions and suggestions. I am grateful thanks to the Secretary, Mr. R. Panchapakesan, ,and Dr.R. Padmavathy, Principal, Seethalakshmi Ramaswami College, Tiruchirappalli - 620 002, for having given me an opportunity to 
do research with the necessary infrastructure and resources to carry out my work. I extend my gratitude to Dr.R.Vasuki, Head, Department of Chemistry, Vice Principal, and SF-In Charge of Seethalakshmi Ramaswami College, Tiruchirappalli - 620 002, for constant encouragement and moral support.

\section{REFERENCES}

[1] Mckinnon, J.J.; Mitchell, A.S; Spackman, M.A, Chem.- Eur. J. 1998, 11, 2136-2141. b) Spackman, M.; Mckinnon, J.J. CrystEng- Comm., 2002, 4, 378-392. c) Mckinnon, J. J.; Spackman, M. A.; Mitchell, A.S, Cryst Crystallogr., Sect. B., 2004, 60, 627-668.

[2] Foster, R. Organic Charge transfer complexes, Academic Press, London 1969.

[3] Mulliken, R.S, J. Am. Chem. Soc., 1952, 74, 811.

[4] Hirshfeld, F.L,Theor. Chim. Acta. 1977, 44, 129.

[5] Spackman, M.A. and McKinnon, J.J, CrystEngComm., 2002, 4, 378.

[6] Wolff, S.K., Grimwood, D.J., McKinnon, J.J., Turner, M.J., Jayatilaka, D. and Spackman, M.A. CrystalExplorer 3.1. University of Western Australia Crawley, Western Australia, 2013.

[7] Allen, F.H., Kennard, O., Watson, D.G., Brammer, L., Orpen, A.G. and Taylor, R. J. Chem. Soc. Perkin Trans., 1987, 2, S1.

[8] Spackman, M.A. and Jayatilaka, D.,CrystEngComm. s2009, 11, 19.

[9] Meyer, A. Y., Chem. Soc. Rev., 1986, 15, 449.

[10]Rudnick, J. and Gaspari, G., J. Phys. A: Math. Gen. Phys., 1986, 19, L191.

[11] Baumgartner, A., J. Chem. Phys., 1993, 99, 7496.

Citation: M.Bhavya \& R.Malarvizhi "Fingerprint Intermolecular Interactions of Sodium and Potassium Complexes, International Journal of Advanced Research in Chemical Science, vol. 7, no. 9, pp. 19-30, 2021. Available: DOI: http://dx.doi.org/ 10.20431 /2454-9444.0709003

Copyright: () 2021 Authors. This is an open-access article distributed under the terms of the Creative Commons Attribution License, which permits unrestricted use, distribution, and reproduction in any medium, provided the original author and source are credited. 